\title{
Tratamento do pterígio recidivado por transplante autólogo de conjuntiva
}

\section{Treatment of recurrent pterygium using conjunctivalautograft}

\author{
Jorge Taveira Samahá ${ }^{1}$ \\ Silvana Artioli Schellini ${ }^{2}$ \\ Regina Hitomi Sakamoto ${ }^{1}$ \\ Carlos Roberto Padovani ${ }^{3}$
}

\section{RESUMO}

Objetivo: Avaliar a resposta ao tratamento do pterígio recidivado, usando a técnica do transplante autólogo de conjuntiva. Métodos: Foi realizado estudo retrospectivo de 36 pacientes ( 36 olhos), portadores de pterígio recidivado, submetidos a transplante autólogo de conjuntiva. O tempo de seguimento mínimo foi de seis meses. Resultados: Observou-se recidiva em $41,6 \%$ dos pacientes, perda do enxerto em 5,5\% e deiscência de sutura em $2,7 \%$. Conclusões: $O$ transplante de conjuntiva é procedimento com baixo índice de complicações. Porém, quando usado no tratamento do pterígio recidivado, a taxa de recorrência permanece alta.

Descritores: Pterígio; Recidiva; Transplante autólogo; Conjuntiva/transplante; Mitomicina/ uso terapêutico; Mitomicina/administração \& dosagem; Seguimentos

\section{INTRODUÇ̃̃O}

Existem vários tipos de tratamento e diferentes modalidades cirúrgicas para a abordagem terapêutica do pterígio, dentre as quais destacam-se: excisão simples ("esclera nua"), excisão com rotação de retalho, excisão com ceratoplastia lamelar, transplante autólogo de conjuntiva e transplante de membrana amniótica ${ }^{(1)}$. O problema maior do tratamento cirúrgico do pterígio é a recidiva do tecido fibrovascular que chega até $89,8 \%$ para alguns procedimentos e tende a ser mais agressiva que a lesão primária ${ }^{(2-3)}$.

Para diminuir as recorrências, à cirurgia pode-se associar tratamento adjuvante como betaterapia ou antimetabólicos (Mitomicina $\mathrm{C}$ ou 5-Fluouracil) ${ }^{(3-5)}$. No entanto, também a introdução de tratamento adjuvante pode estar relacionada a complicações, algumas de significativo grau de severidade ${ }^{(6-7)}$.

O transplante autólogo de conjuntiva é uma técnica que foi popularizada a partir de 1985 como método efetivo para reduzir as taxas de recidiva do pterígio. Esta técnica consiste no enxerto de conjuntiva livre, obtido do quadrante súpero-temporal do mesmo olho, para recobrir a esclera exposta após a excisão da lesão. Kenyon et al. observaram recorrência de 5,3\% em pterígios avançados ou recidivados ${ }^{(8)}$. No entanto, taxas maiores de recidiva foram detectadas em áreas geográficas com maior exposição à radiação ultravioleta ${ }^{(9-11)}$.

Conduziu-se o presente estudo com o objetivo de avaliar a taxa de recidiva e a ocorrência de complicações em pacientes com pterígio recidivado, submetidos ao transplante autólogo de conjuntiva em nosso serviço.

\section{MÉTODOS}

Foi realizado estudo retrospectivo em 36 olhos de 36 pacientes com pterígio recidivado que foram submetidos a transplante autólogo de conjun- 
tiva, no período entre janeiro de 1997 e outubro de 1999. Foram avaliados: idade, sexo, localização, olho acometido, número de cirurgias anteriores, uso prévio de antimetabólicos, existência de complicações e a presença ou não de recidiva, com tempo mínimo de seguimento pós-operatório de seis meses.

Técnica Cirúrgica: a técnica utilizada foi similar à descrita por Kenyon et al. ${ }^{(7)}$, com modificações feitas por Allan et al..$^{(10)}$ : 1) injeção subconjuntival de lidocaína $2,0 \%$ com vasoconstritor, com olhar direcionado de forma a expor o campo cirúrgico; 2) dissecção da "cabeça" do pterígio com bisturi lâmina 15 ; 3 ) cauterização minimizada, procurando preservar a vascularização do leito do enxerto; 4) ressecção da "cabeça" e porção do "corpo" com tesoura de conjuntiva; 5) delimitação do tamanho da área receptora com compasso cirúrgico; 6) delimitação da área doadora no quadrante súpero-temporal, $1,5 \mathrm{~mm}$ posteriormente ao limbo; 7) infiltração de anestésico subconjuntival no leito doador; 8) remoção do enxerto com tesoura de conjuntiva, excluindo-se a cápsula de Tenon; 9) a conjuntiva foi transportada para o leito receptor, respeitando-se a orientação limbo/limbo da conjuntiva a ser transplantada com a área receptora; 10) fixação do enxerto na área receptora por meio de dois pontos separados na episclera límbica às 14 e 16 horas, seguindo-se por pontos contínuos usando-se fio de Vicryl 7-0.

Ao final da cirurgia, foi instilado colírio de neomicina, polimixina B e dexametasona e realizado curativo oclusivo a ser mantido por 24 horas. No pós-operatório, utilizou-se o mesmo colírio quatro vezes por dia, durante 21 dias. As cirurgias foram realizadas por residentes de $2^{\circ} \mathrm{e} 3^{\circ}$ ano.

Os pacientes foram avaliados quanto à presença de recidiva clínica, caracterizada por tecido fibroso e vascular atingindo a superfície corneana e passando além do limbo ou presença de complicações.

Os dados obtidos foram submetidos à análise estatística, usando o Teste de Goodman.

\section{RESULTADOS}

Dos 36 pacientes estudados, 19 possuíam pterígio no olho direito e 17 no olho esquerdo. $47,2 \%$ eram masculinos e $53,8 \%$ femininos. A idade média dos pacientes foi $47,8 \pm 29,2$ anos, variando de 22 a 79 anos. Dezoito pacientes (50,0\%) encontravam-se na faixa etária de 40 a 59 anos, 10 pacientes $(27,7 \%)$ entre 20 a 39 anos e $8(22,3 \%)$ eram maiores que 60 anos. Não houve diferença estatisticamente significativa entre os grupos de pacientes que apresentaram ou não recidiva clínica, com relação à idade $(p>0,05)$. Não se encontrou, também, diferença estatisticamente significativa quanto aos fatores sexo, olho acometido, uso prévio ou não de anti-metabólitos, entre os pacientes que apresentaram ou não recidiva do pterígio.

Vinte e quatro pacientes $(66,6 \%)$ haviam sido submetidos a um procedimento cirúrgico prévio, e 12 (33,3\%), submetidos a dois procedimentos anteriores. Nos 11 pacientes $(30,5 \%)$ que usaram previamente os anti-metobólitos, o medicamento utilizado foi o 5-Fluorouracil.
Observou-se no pós-operatório as seguintes complicações: perda do enxerto em 2 casos $(5,55 \%)$ e 1 paciente com deiscência da sutura $(2,77 \%)$. Esses 3 casos, evoluíram com recidiva 2, 3 e 6 de meses após a cirurgia, respectivamente.

A taxa de recorrência foi de 41,6\% (15 casos), com intervalo de tempo entre o transplante de conjuntiva e a recidiva variando de 1 até mais do que 6 meses (Tabela 1).

\section{DISCUSSÃO}

As várias técnicas disponíveis para o tratamento do pterígio devem ser avaliadas com referência a dois critérios principais: segurança (ausência de complicações que comprometam a visão) e eficácia (ausência de recidiva).

As complicações decorrentes do transplante autólogo de conjuntiva foram infreqüentes no presente estudo e não necessitaram de reoperação. Não foram observadas complicações como granuloma da Tenon, cisto conjuntival, infeção ou falência do transplante de conjuntiva ${ }^{(12-13)}$. Porém, $41,6 \%$ dos pacientes evoluíram com presença de nova recidiva que é o principal problema na abordagem do pterígio. Apesar de termos observado $41,6 \%$ de recidiva, o transplante autólogo de conjuntiva tem sido reportado como uma técnica com baixas taxas de recorrência (Quadro 1) ${ }^{(2,8,14-15)}$.

A taxa de recidiva pode ser influenciada por: fatores relacionados com a resposta do hospedeiro, variações da técnica empregada, uso de terapia adjuvante, diferentes medicações utilizadas no pós-operatório, localização geográfica da população estudada, a duração do período de seguimento e pela definição de recorrência empregada ${ }^{(12)}$.

Cunha et al. ${ }^{(14)}$, em portadores de pterígio primário e recorrente, encontraram taxa de recidiva de $4,16 \%$, sendo a recidiva observada apenas entre os portadores de pterígios já recidivados. No estudo conduzido por Garcia et al. ${ }^{(15)}$, também em portadores de pterígio primário e recidivado, não se observou nenhum caso de recidiva. Allan et al. ${ }^{(12)}$, encontraram taxa de recidiva de $6,8 \%$, com apenas 1 caso de recidiva e que ocorreu entre os pterígios primários. Riordan-Eva et al. ${ }^{(2)}$, encontraram taxa de recidiva de $62,0 \%$ em pacientes operados pela técnica da "esclera nua", contra $13,0 \%$ nos submetidos ao transplante autólogo de conjuntiva em pacientes com pterígio primário e $7,0 \%$, para pacientes com pterígio recorrente. Figueiredo et al. ${ }^{(16)}$

\begin{tabular}{|c|c|c|}
\hline \multicolumn{3}{|c|}{$\begin{array}{c}\text { Tabela 1. Distribuição das recidivas observadas após a } \\
\text { realização do transplante de conjuntiva autólogo segundo } \\
\text { época de ocorrência }\end{array}$} \\
\hline Recidiva & $n^{\circ}$ pacientes & $\%$ \\
\hline até 1 mês & 2 & 13,3 \\
\hline 1 a 3 meses & 3 & 20,0 \\
\hline 3 a 6 meses & 3 & 20,0 \\
\hline mais que 6 meses & 7 & 46,6 \\
\hline Total & 15 & 100,0 \\
\hline
\end{tabular}


Quadro 1. Taxas de recidiva após transplante autólogo de conjuntiva, segundo autor localidade e tipo de amostragem do estudo

\begin{tabular}{|c|c|c|c|c|}
\hline Autor & Localidade & Tipo de estudo & $n^{0}$ de casos & Taxa de recidiva (\%) \\
\hline Allan et al., 1993 & Austrália & Retrospectivo & 93 & $6,8 \mathrm{PP}$ \\
\hline Figueiredo et al., 1997 & USA & Retrospectivo & 40 & $16,0 \mathrm{PP}$ \\
\hline Chen et al., 1995 & Singapura & Prospectivo Randomizado & 23 & 39,0 \\
\hline Tan et al.,1997 & Singapura & Prospectivo Randomizado & 61 & 2,0 PP \\
\hline
\end{tabular}

tiveram $16 \%$ de recidiva em pterígios primários, operados pela técnica do transplante de conjuntiva.

Estudos randomizados mostraram resultados discordantes, quando comparadas às técnicas de "esclera nua" e transplante de conjuntiva. Para Tan et al. ${ }^{(17)}$, a taxa de recidiva com a técnica de "esclera nua" foi de $61,0 \%$ contra $2,0 \%$ nos submetidos a transplante de conjuntiva, no caso de pterígio primário e de $82,0 \%$ e zero, respectivamente nos portadores de pterígio recorrente $^{(17)}$. Porém, Lewallen ${ }^{(9)}$ não evidenciou resultados significativamente melhores com o transplante autólogo de conjuntiva sobre a técnica de "esclera nua". Mahar ${ }^{(10)}$ encontrou taxa de recorrência de $25,9 \%$ entre pacientes submetidos a transplante de conjuntiva contra uma taxa de recorrência de $9,4 \%$ nos operados pela técnica da "esclera nua" e que receberam colírio de Mitomicina-C a $0,02 \%$ no pós-operatório. Também usando Mitomicina-C tópica no pós-operatório, Caem et al. ${ }^{(11)}$ estabeleceram taxa de recidiva de $38,0 \%$; sem uso do antimetabólico as taxas variaram entre $39,0 \%$ para o transplante de conjuntiva e $88,0 \%$ para a técnica de "esclera nua".

No presente estudo, as cirurgias foram realizadas por cirurgiões em formação, o que pode influenciar no resultado do tratamento, uma vez que a técnica cirúrgica e o tamanho adequado do enxerto são importantes para conter a recidiva ${ }^{(12-13)}$. O fio absorvível trançado que foi utilizado para a sutura conjuntival promove mais inflamação que os fios de monofilamento, o que também pode atrair neoformação vascular, podendo influir na recidiva.

Apesar da taxa de recidiva observada no presente estudo ser relativamente alta, deve-se lembrar que o transplante de conjuntiva foi utilizado para o tratamento do pterígio previamente recidivado e, portanto, com maiores chances de recidiva.

\section{CONCLUSÃO}

O transplante de conjuntiva é procedimento com baixo índice de complicações. Porém, quando usado no tratamento do pterígio recidivado, a taxa de recorrência permanece alta. $\mathrm{O}$ procedimento cirúrgico é mais demorado e trabalhoso em comparação com outras técnicas. Porém, é livre de complicações potencialmente graves e é uma técnica para a qual não há necessidade de material ou cirurgia complementar, nem de cuidados pós-operatórios especiais.

Devido aos discordantes resultados da literatura com relação à taxa de recidiva, novos estudos clínicos prospectivos e randomizados serão necessários para determinar fatores que possam apresentar influência sobre a eficiência do procedimento, a fim de se determinar a real vantagem do transplante autólogo de conjuntiva sobre as demais técnicas de abordagem cirúrgica do pterígio.

\section{ABSTRACT}

Purpose: To evaluate the use of conjunctival autograft transplantation in the treatment of recurrent pterygium. Methods: Retrospective study of 36 patients (36 eyes), with recurrent pterygium, who underwent conjunctival autograft as a treatment option for recurrent pterygium. The minimal follow up was 6 months. Results: We observed a recurrency rate of $41.6 \%$, $5.5 \%$ had loss of graft and $2.7 \%$ dehiscence. Conclusion: Conjunctival autograph is a safe procedure for recurrent pterygium, with few complications, although, the recurrence rate is still high in these patients.

Keywords: Pterygium; Recurrence; Autologous transplantation; Conjunctiva/transplantation; Mitomycin/therapeutic use; Mitomycin/administration \& dosage; Followup

\section{REFERÊNCIAS}

1. Adamis AP, Starck T, Kenyon DR. The management of pterygium. Ophthalmol Clin North Am. 1990;3:611-23.

2. Riordan-Eva P, Kielhorn I, Ficker LA, Steele AD, Kirkness CM. Conjunctival autografting in the surgical management of pterygium. Eye 1993;7(Pt.5):634-8.

3. Singh G, Wilson MR, Foster CS. Mitomycin eye drops as treatment for pterygium. Ophthalmology 1988;95:813-21.

4. Cardillo JA, Alves MR, Kara-José N, Tranjan-Neto A, Serpa JF, Ambrosio LE. Uso tópico pós-operatório versus aplicação intra-operatória da mitomicina-C a $0,02 \%$ na prevenção de recidivas pós-operatórias do pterígio primário. Arq Bras Oftalmol 1995;58:413-5.

5. Shiratori CN, Spirandelli PH, Shiratori C, Schellini AS, Padovani CR. Uso do 5-fluouracil intra-operatório na cirurgia do pterígio. Arq Bras Oftalmol 1999;62:501-4. 
6. Rubinfeld RS, Pfister RR, Stein RM, Foster CS, Martin NF, Stoleru S, et al Serious complications of topical mitomycin-C after pterygium surgery [commented on Ophthalmology 1992;99:1645-6]. Ophthalmology 1992;99:1647-54.

7. Kato E, Macruz E, Alves MR. Complicação ocular grave após ressecção de pterígio e uso de colírio de mitomicina-C. Relato de dois casos. In: Kara-José N, Alves MR. Conjuntiva cirúrgica. São Paulo: Roca;1999. p.107-11.

8. Kenyon KR, Wagoner MD, Hettinger ME. Conjunctival autograft transplantation for advanced and recurrent pterygium. Ophthalmology 1985;92:1461-70.

9. Lewallen S. A randomized trial of conjunctival autografting for pterygium in the tropics. [commented on Ophthalmology 1990;97:542-3]. Ophthalmology 1989;96:1612-4.

10. Mahar PS. Conjunctival autograft versus topical mitomycin-C in treatment of pterygium. Eye 1997;11(Pt 6):790-2.

11. Chen PP, Ariyasu RG, Kaza V, LaBree LD, McDonell PJ. A randomized trial comparing mitomycin-C and conjunctival autograft after excision of primary pterygium [commented on Am J Ophthalmol 1996;121:333-4]. Am J Ophthalmol 1995;120:151-60.
12. Allan BDS, Short P, Crawford GJ, Barret GD, Constable IJ. Pterygium excision with conjunctival autografting: an effective and safe technique [commented on $\mathrm{Br}$ J Ophthalmol 1994;78:421]. Br J Ophthalmol 1993;77:698-701.

13. Starck T, Kenyon KR, Serrano F. Conjunctival autograft for primary and recurrent pterygia: surgical technique and problem management. Cornea 1991;10:196-202.

14. Cunha M, Allemann N. Transplante autólogo de conjuntiva no tratamento de pterígio primário e recidivado. Arq Bras Oftalmol 1993;56:78-81.

15. Garcia SG, Alleoni S, Junqueira AM, Rezende R. Técnica de enxerto autólogo de conjuntiva em pterigiectomia primária e recidivada. Rev Bras Oftalmol 1994;53:277-80.

16. Figueiredo RS, Cohen EJ, Gomes JA, Rapuano CJ, Laibson PR. Conjunctival autograft for pterygium surgery: how well does it prevent recurrence? [commented on Ophthalmic Surg Lasers 1997;28:875-7]. Ophthalmic Surg Lasers 1997;28:875-7.

17. Tan DT, Chee SP, Dear KB, Lim AS. Effect of pterygium morphology on pterygium recurrence in a controlled trial comparing conjunctival autografting with bare sclera excision. [published erratum Arch Ophthalmol 1998;116:552]. Arch Ophthalmol 1997;115:1235-40.

\title{
WORKSHOP DE RETINOPATIA DA PREMATURIDADE
}

\author{
04 a 06 de Outubro de 2002
}

\author{
Anfiteatro do Instituto Brasileiro de Oftalmologia \\ Rio de Janeiro - RJ
}

\section{Promoção: \\ Agência Internacional da Prevenção da Cegueira (IAPB)}

- CBM International

- Instituto VIDI

- Conselho Brasileiro de Oftalmologia

INFORMAÇÕES: tel.: (11) 287-9217 (Sra. Regina Alcoba)

e-mail: aazin@terra.com.br 